Journal of Finance Research

\title{
The Impact of Cost Information Sharing on Procurement Contract Design
}

\section{Chao Dong* Yankang Chen}

Huazhong University of Science and Technology, Wuhan, Hubei, 430074, China

\begin{tabular}{|c|c|}
\hline ARTICLE INFO & ABSTRACT \\
\hline $\begin{array}{l}\text { Article history } \\
\text { Received: } 16 \text { June } 2020 \\
\text { Revised: } 23 \text { June } 2020 \\
\text { Accepted: } 9 \text { October } 2020 \\
\text { Published Online: } 16 \text { October } 2020 \\
\text { Keywords: } \\
\text { Information asymmetry } \\
\text { Mechanism design } \\
\text { Supply chain outsourcing }\end{array}$ & $\begin{array}{l}\text { In this paper, we provides contract design mechanisms and analysis for } \\
\text { manufacturers to manage decentralized supply chain. Suppose the man- } \\
\text { ufacturer's final product consists of components, each produced by a } \\
\text { different supplier, and the manufacturer first purchases components from } \\
\text { suppliers, then assembles them into final product and meet demands af- } \\
\text { termarket realization. While supply chain's internal cooperation always } \\
\text { benefits both, suppliers are often reluctant to proactively share their own } \\
\text { production cost structure, otherwise manufacturers may depress purchase } \\
\text { prices, which may reduce supplier's profit. Manufacturers on the other } \\
\text { hand, prefers to be informed of true cost information in order to gain } \\
\text { greater revenues. We takes manufacturer's perspective and design the } \\
\text { optimal contract menu for suppliers, both to enable suppliers to disclose } \\
\text { private cost information and to maximize the benefits. We start by mod- } \\
\text { eling the original problem and find that the original problem is a complex } \\
\text { multidimensional optimization problem. We then examine the nature of } \\
\text { the original problem solving and devise the solution algorithm to arrive at } \\
\text { the optimal contract menu. This algorithm reduces the complexity of the } \\
\text { original question from o( } 2 \mathrm{n} \text { ) to o(n). We further investigate the influence } \\
\text { mechanism of model parameters on the results and find that when market } \\
\text { demand increases or the selling price of the final product increases, value } \\
\text { of private information increases significantly. However, if market demand } \\
\text { uncertainty increases, the value of information may increase or decrease } \\
\text { for both sides. }\end{array}$ \\
\hline
\end{tabular}

\footnotetext{
*Corresponding Author:

Chao Dong,

Male, undergraduate,

Research direction: logistics management;

Correspondence address: Huazhong University of Science and Technology, Wuhan City,Hubei Province, 430074, China;

E-mail:2556354763@qq.com.

Yankang Chen,

Male, undergraduate,

Research direction: logistics management;

Correspondence address: Huazhong University of Science and Technology, Wuhan City,Hubei Province, 430074, China;

E-mail:754932869@qq.com.
} 


\section{Introduction}

$\mathrm{A}$ s the complexity of supply chain grows continuously, it is insufficient for big manufacturers to satisfies their demand completely by their own. For example, Ford outsources $65 \%$ of its own components, General Motors outsources over 55\% and Crysler over $80 \%{ }^{[1]}$. In general, big manufacturer have plenty of upstream suppliers. Coordination with suppliers is the major challenge constantly faced by big firms.

Information sharing refers to the sharing of private information between supply chain enterprises to achieve upstream and downstream cooperation and enhance the overall effectiveness of the supply chain. Many branches in the logistics field can collaborate effectively by sharing information. Information sharing between suppliers and manufacturers allows both parties to make optimal production and replenishment strategies, reduce supply chain uncertainty, reduce overall costs, and improve customer service. The information shared between companies can be divided into the following categories: inventory information, sales information, and demand forecast information, order status information, production planning information, etc. Among them, inventory information and production planning information is two-way shared by upstream and downstream enterprises, sales information and demand forecast information are generated by manufacturers and shared with upstream suppliers. In contrast, order status information is held by suppliers in real-time and shared with manufacturers. Also, the information available for sharing includes product quality information, new delivery information, etc.

As information technology continues to evolve, information sharing has also become faster and more efficient ${ }^{[2]}$. However, information asymmetries persist, and the risks they pose remain insurmountable. Each enterprise within the supply chain has its ideas about operational mechanisms, so the objectives often conflict with those of the supply chain as a whole. Low visibility of information between companies will lead to a waste of resources and underneath the overall efficiency of the supply chain. The management of uncertainty risks and coordination mechanisms have become essential breakthroughs in the optimization of supply chain systems ${ }^{[3]}$. They are reducing uncertainty through information sharing to promote closer strategic coordination and cooperation to jointly address the challenges to supply chains posed by information asymmetry and the risk of uncertainty.

The main purpose of manufacturers outsourcing some components to upstream suppliers is to minimize the costs associated with maintaining product lines, since many of these components are too costly while in low demand ${ }^{[4-5]}$. Outsourcing production can increase production integration and reduce overall cost. In reality, however, sometimes outsourcing does not reduce costs, but rather could lead to cost increase ${ }^{[6]}$. This is because outsourcing creates a decentralized supply chain, where suppliers and manufacturers are financially independent. Therefore, they both seek their best interests. On this basis, both parties tend not to share private information before come into a cooperation plan, as any sharing of private information may result in losing some of its bargaining power ${ }^{[7]}$, which is not conducive to maximizing its benefits.

In this paper, manufacturers with greater bargaining power are faced with a number of factors to consider when developing a coordination strategy for outsourcing production. First, manufacturers tend to obtain accurate private supplier information, i.e., information on cost structure, product quality, etc., in order to keep the price paid as low as possible in order to obtain a greater profit. At the same time, manufacturers should also consider whether suppliers will report false information, which may result in additional cost. Outsourced, decentralized supply chain reduces transparency, and asymmetries in cost information ultimately reduce manufacturer profitability. It is therefore particularly important to establish contractual design mechanisms in such supply chain that facilitate collaboration between the parties and reduce the impact of information asymmetries.

The conflict of interest between suppliers and manufacturer belongs to the ex ante asymmetric information game, which satisfies the reverse choice model in Principal-agent Theory. The manufacturer is the client and has greater bargaining power, so that manufacturer only needs to offer contracts from its own point of view, without further negotiation with the supplier; Suppliers are agents and have information about production costs, production quality, etc, which is unknown to the manufacturer. Manufacturer provides a list of contracts on quantities to be purchased and price to be paid, based on a subjective judgment of the supplier's cost structure, and the supplier decides whether accept this contract or not to begin production cooperation. This paper applies the theory of mechanism design to provide manufacturers with contract design solutions. This paper will focus on how a downstream manufacturer, facing with uncertain cost information, can overcome conflicts of interest between the parties and design ideal contract models.

\section{Modeling Framework}

We consider a two-echelon supply chain system with $n$ suppliers (she) and 1 manufacturer (he). Manufacturer 
procures component from suppliers in order to assemble final products. Each final product requires one component from each supplier. Suppliers are irreplaceable from each other. Manufacturer pays after receiving goods.

Supply chain faces uncertain market demand $D$, and the final product can be sold at fixed price $r$. We assume supplier $i$ incurs a unit production $\operatorname{cost} c_{i}, i=1, \ldots . n$, which is private information for the supplier only. The manufacturer only has a subjective assessment about each supplier's cost.

Manufacturer believes cost from each supplier falls into $c_{i}^{H}$ (high type) or $c_{i}^{L}$ (low type), with a joint probability of $P\left(c_{i}=c_{i}^{t_{i}}, \forall i=1, \ldots, n, t_{i}=H, L\right)$ and $c_{i}^{H} \geq c_{i}^{L}$, the probability density function is $p_{i}^{k}=P\left(c_{i}=c_{i}^{k}\right), k=H, L, \forall i=1, \ldots, n, p_{i}^{H}+p_{i}^{L}=1$. We assume the sum of components' cost no greater than final product price,i.e., $\sum_{i=1}^{n} c_{i}^{H}<r$ in order to guarantee manufacturer's profitability.

According to the principle of mechanism design, manufacturer maximizes his profit by providing optimal menu of contracts to each supplier ${ }^{[8]}$. Based on revelation principle, contracts offered by manufacturer should be incentive compatible. For each supplier has two types of cost, manufacturer offers two separate contracts, $\left(Q_{i}^{H}, X_{i}^{H}\right),\left(Q_{i}^{L}, X_{i}^{L}\right)$ . If supplier $i$ with $c_{i}=c_{i}^{t_{i}}\left(t_{i}=H, L\right)$ chooses contract $\left(Q_{i}^{k}, X_{i}^{K}\right)$, where $k=L, H$, then she agrees to produce and deliver $Q_{i}^{k}$ units of his component to the manufacturer in return for a total payment of $X_{i}^{k}$. Supplier $i$ 's profit is $X_{i}^{k}-c_{i}^{t_{i}} Q_{i}^{k}$. After manufacturer realizes actual demand, he assembles components into final product and satisfy the market as far as he can ${ }^{[9]}$.

Manufacturer's problem can be formulated into the following model:

$\max _{\substack{\left(X_{i}^{H}, Q_{i}^{H}\right),\left(X_{i}^{L}, Q_{i}^{L}\right) \\ i=1, \ldots, n}} \Pi=E_{D}\left\{\sum_{\substack{t_{i}=H, L \\ i=1, \ldots, n}} P\left(c_{i}=c_{i}^{t_{i}}, \forall i=1, \ldots, n\right)\left[r \min \left(Q_{1}^{t_{1}}, \ldots, Q_{n}^{t_{n}}, D\right)-\sum_{i=1}^{n} X_{i}^{t_{i}}\right]\right\}$

$$
\text { s.t. }=\left\{\begin{array}{l}
(\text { I.C.High }) X_{i}^{H}-c_{i}^{H} Q_{i}^{H} \geq X_{i}^{L}-c_{i}^{H} Q_{i}^{L}, i=1, \ldots, n \\
(\text { I.C.Low }) X_{i}^{L}-c_{i}^{L} Q_{i}^{L} \geq X_{i}^{H}-c_{i}^{L} Q_{i}^{H}, i=1, \ldots, n \\
(\text { I.R.High }) X_{i}^{H}-c_{i}^{H} Q_{i}^{H} \geq 0, i=1, \ldots, n \\
(\text { I.R.Low }) X_{i}^{L}-c_{i}^{L} Q_{i}^{L} \geq 0, i=1, \ldots, n
\end{array}\right.
$$

While each supplier has two potential contracts, manufacturer has $2^{n}$ possible revenue outcome. For instance, when there's two supplier $(n=2)$, manufacturer's profit function $\Pi$ can be written as follows:

$$
\begin{aligned}
\Pi= & \mathrm{E}\left\{P\left(c_{1}=c_{1}^{H}, c_{2}=c_{2}^{H}\right)\left[\operatorname{rmin}\left(Q_{1}^{H}, Q_{2}^{H}, D\right)-x_{1}^{H}-x_{2}^{H}\right]\right. \\
& +P\left(c_{1}=c_{1}^{H}, c_{2}=c_{2}^{L}\right)\left[\operatorname{rmin}\left(Q_{1}^{H}, Q_{2}^{L}, D\right)-x_{1}^{H}-x_{2}^{L}\right] \\
& +P\left(c_{1}=c_{1}^{L}, c_{2}=c_{2}^{H}\right)\left[\operatorname{rmin}\left(Q_{1}^{L}, Q_{2}^{H}, D\right)-x_{1}^{L}-x_{2}^{H}\right] \\
& \left.+P\left(c_{1}=c_{1}^{L}, c_{2}=c_{2}^{L}\right)\left[\operatorname{rmin}\left(Q_{1}^{L}, Q_{2}^{L}, D\right)-x_{1}^{L}-x_{2}^{L}\right]\right\}
\end{aligned}
$$

There are $4 n$ constraints for this model. Incentive Compatibility constraints (I.C.High),(I.C.Low) ensure each supplier choose optimal contract based on their true cost type. Individual Rationality Constraints (I.R.High),(I.R.Low) ensures supplier can have non-negative profit, which prevents interruption of cooperation.

\section{Contract design under different informa- tion transparency}

\subsection{Benchmark: symmetric cost information}

Under symmetric information, the cost type would be revealed to manufacturer and supplier herself simultaneously. Therefore, manufacturer could know supplier $i$ 's cost $t_{i}$ precisely, and provides her with contract $\left(Q_{i}^{t_{i}}, X_{i}^{t_{i}}\right)$, supplier can either accept this contract or reject it. Hence, the incentive compatibility constraints no longer exist. For given realization of supplier's costs, i,e., $c_{i}=c_{i}^{t_{i}}, \forall i=1, \ldots, n$ , the manufacturer's problem writes as follows:

$$
\begin{array}{r}
\max \tilde{\pi}\left(Q_{i}^{t_{i}}, X_{i}^{t_{i}}\right)=r \operatorname{Emin}\left(Q_{1}^{t_{1}}, \ldots, Q_{n}^{t_{n}}, D\right)-\sum_{i=1}^{n} X_{i}^{t_{i}} \\
\text { s.t.(I.R.) } X_{i}^{t_{i}}-c_{i}^{t_{i}} Q_{i}^{t_{i}} \geq 0, \forall i=1, \ldots, n
\end{array}
$$

Manufacturer's revenue function $\tilde{\pi}$ decreases while $X_{i}^{t_{i}}$ increase, therefore manufacturer obtains maximum revenue when $X_{i}^{t_{i}}=c_{i}^{t_{i}} Q_{i}^{t_{i}}$. The function can be reformulated as

$$
\max \tilde{\pi}\left(Q_{i}^{t_{i}}, X_{i}^{t_{i}}\right)=r \operatorname{Emin}\left(Q_{1}^{t_{1}}, \ldots, Q_{n}^{t_{n}}, D\right)-\sum_{i=1}^{n} c_{i}^{t_{i}} Q_{i}^{t_{i}}
$$

It can be observed that manufacturer profit is restricted by minimum procurement quantity from suppliers. Thus the manufacturer would procure the exact same quantity of each components from suppliers, i.e. $Q_{1}^{t_{1}}=\ldots=Q_{n}^{t_{n}}=Q$. We derive the function and it is straightforward to show the following result:

Lemma 1 For any given $c_{i}=c_{i}^{t_{i}}, i=1, \ldots, n$, the manufacturer's optimal contract is 


$$
\forall i=1, \ldots, n, Q_{i}^{t_{i}}=\bar{F}^{-1}\left(\frac{\sum_{i=1}^{n} c_{i}^{t_{i}}}{r}\right), X_{i}^{t_{i}}=c_{i}^{t_{i}} Q_{i}^{t_{i}}
$$

Suppliers always get zero profit under symmetric information, and the manufacturer captures entire profit. The expected optimal profit function is

$$
\tilde{\Pi}=\sum_{\substack{t_{i}=H, L \\ i=1, \ldots, n}} P\left(c_{i}=c_{i}^{t_{i}}, \forall i=1, \ldots, n\right) \tilde{\pi}\left(Q_{i}^{t_{i}}, X_{i}^{t_{i}}\right)
$$

\subsection{Asymmetric cost information}

In this section, we continue to design the list of contracts under asymmetric cost information. According to standard analysis for mechanism design problems, we can show that constraints (I.R.High) and (I.C.Low) must be binding at optimality, i.e.,

$X_{i}^{H}=c_{i}^{H} Q_{i}^{H}$

$$
X_{i}^{L}=Q_{i}^{H}\left(c_{i}^{H}-c_{i}^{L}\right)+c_{i}^{L} Q_{i}^{L}
$$

Furthermore, it follows that (I.C.Low), $c_{i}^{H} \geq c_{i}^{L}$, and (I.R.High) that constraint (I.R.Low) is redundant. Substituting $X_{i}^{H}$ and $X_{i}^{L}$ above into (I.C.High), it follows directly that (I.C.High) is automatically satisfied if $Q_{i}^{H} \leq Q_{i}^{L}, \forall i=1, \ldots, n$. The manufacturer's problem given by (1) reduces to the following problem

$$
\begin{gathered}
\max _{\substack{Q_{i}^{H}, Q_{i}^{L} \\
i=1, \ldots, n}} \quad \Pi=\sum_{\substack{i=1, \ldots, n \\
t_{i}=H, L}}\left[P\left(c_{i}=c_{i}^{t_{i}}, \forall i=1, \ldots, n\right) r \operatorname{Emin}\left(Q_{1}^{t_{1}}, \ldots, Q_{n}^{t_{n}}, D\right)\right] \\
\quad-\sum_{i=1}^{n}\left(c_{i}^{H}-p_{i}^{L} c_{i}^{L}\right) Q_{i}^{H}-\sum_{i=1}^{n} p_{i}^{L} c_{i}^{L} Q_{i}^{L}
\end{gathered}
$$

subject to

$$
Q_{i}^{H} \leq Q_{i}^{L}, i=1, \ldots, n
$$

We denote $\vec{Q}=\left(\left(Q_{1}^{H}, Q_{1}^{L}\right), \ldots,\left(Q_{n}^{H}, Q_{n}^{L}\right)\right)$ as the optimal solution to problem (7).

\subsubsection{1-Supplier Situation}

We first solve a simple case of $n=1$, which means there exists only one supplier. The partial derivative of $Q_{1}^{H}$ and $Q_{1}^{L}$ from the manufacturer's profit function can be written as follows

$$
\begin{gathered}
\frac{\partial \Pi}{\partial Q_{1}^{H}}=p_{1}^{H} r \bar{F}\left(Q_{1}^{H}\right)-\left(c_{1}^{H}-p_{1}^{L} c_{1}^{L}\right) \\
\frac{\partial \Pi}{\partial Q_{1}^{L}}=p_{1}^{L} r \bar{F}\left(Q_{1}^{L}\right)-p_{1}^{L} c_{1}^{L}
\end{gathered}
$$

Manufacturer's revenue would decrease while order quantities increase, because $\bar{F}$ is a non-increasing function. Under the low-cost type, the manufacturer's optimal procurement quantity is $Q_{1}^{L}=\bar{F}^{-1}\left(\frac{c_{1}^{L}}{r}\right)$. When the cost type is high, the optimal procurement quantity is $Q_{1}^{H}=\bar{F}^{-1}\left(\frac{c_{1}^{H}-p_{1}^{L} c_{1}^{L}}{p_{1}^{H} r}\right)$ if $c_{1}^{H}-p_{1}^{L} c_{1}^{L}<p_{1}^{H} r$ is satisfied. Or else, $Q_{1}^{H}=0$.

\subsubsection{N-Suppliers Situation}

We expand the scale of supplier from 1 to $n$. First, we define high cost type procurement quantity for each supplier $i$ as:

$$
q_{i}^{0} \stackrel{\operatorname{def}}{=}\left\{\begin{array}{l}
\bar{F}^{-1}\left(\frac{c_{i}^{H}-p_{i}^{L} c_{i}^{L}}{p_{i}^{H} r}\right), c_{i}^{H}-p_{i}^{L} c_{i}^{L}<p_{i}^{H} r \\
0, \text { otherwise }
\end{array}\right.
$$

Recall that $q_{i}^{0}$ is supplier $i$ 's optimal production quantity if supplier $i$ is a sole supplier in the system and the cost type is high. Without loss of generality, we number the suppliers such that

$q_{1}^{0} \leq q_{2}^{0} \leq \ldots \leq q_{n}^{0}$

We can now present an theorem as follows

Theorem 1 The optimal solution to problem (7) satisfies

$$
Q_{1}^{H} \leq Q_{2}^{H} \leq \ldots \leq Q_{n}^{H} \leq Q_{1}^{L}=Q_{2}^{L}=\ldots=Q_{n}^{L}
$$

We define $Q^{L}=Q_{j}^{L}, \forall j=1, \ldots, n$.

Theorem 1 provides with an important feature for problem solving, for it reduces variable amount from $2 n$ to $n+1$.

For notation convenience, for $1 \leq i \leq j \leq n+1$, we define

$$
\alpha_{i, j}=\left\{\begin{array}{c}
\sum_{\substack{d=i \\
j}}^{j}\left(c_{d}^{H}-p_{d}^{L} c_{d}^{L}\right), i f j \leq n \\
\sum_{d=1}^{i-1} p_{d}^{L} c_{d}^{L}+\sum_{d=i}^{n} c_{d}^{H}, i f j=n+1
\end{array}\right.
$$




$$
\beta_{i, j} \stackrel{\operatorname{def}}{=}\left\{\begin{array}{c}
P\left(c_{b}=c_{b}^{L}, \forall 1 \leq b \leq i-1\right)-P\left(c_{b}=c_{b}^{L}, \forall 1 \leq b \leq j\right), i f j \leq n \\
P\left(c_{b}=c_{b}^{L}, \forall 1 \leq b \leq i-1\right), i f j=n+1
\end{array}\right.
$$

We are able to decompose the problem (7) into a summation of $n+1$ functions with a single variable as follows:

Lemma 2 Problem (7) is equivalent to the following problem:

$$
\begin{gathered}
\max _{0 \leq Q_{1}^{H} \leq Q_{2}^{H} \leq \ldots \leq Q_{n+1}^{H}} \sum_{i=1}^{n+1} M_{i, i}\left(Q_{i}^{H}\right) \\
\text { where } Q_{n+1}^{H} \stackrel{\text { def }}{=} Q^{L} \text { and } \\
M_{i, i}(Q) \stackrel{\operatorname{def}}{=} r \beta_{i, i} \int_{0}^{Q} \bar{F}(x) d x-\alpha_{i, i} Q
\end{gathered}
$$

Further study on $M_{i, i}(Q)$ enables us to establish the following result:

Lemma $3 \forall i, j, 1 \leq i \leq j \leq n+1$, we define functions

$$
\begin{gathered}
M_{i, j}(Q) \stackrel{\text { def }}{=} r \beta_{i, j} \int_{0}^{Q} \bar{F}(x) d x-\alpha_{i, j} Q \\
M_{i, j}(Q)=\sum_{d=i}^{j} M_{d, d}(Q) . M_{i, j}(Q) \text { reaches its maximum at }
\end{gathered}
$$

$Q_{i, j}^{*}$ where if $1 \leq j \leq n$, then

$$
Q_{i, j}^{*}=\left\{\begin{array}{c}
\bar{F}^{-1}\left(\frac{\alpha_{i, j}}{r \beta_{i, j}}\right), \text { if } \alpha_{i, j}<r \beta_{i, j} \\
0, \text { otherwise }
\end{array}\right.
$$

\subsubsection{Algorithm Design}

We design an specific algorithm to solve this problem. It can be proved that $Q_{1}^{H}<Q_{2}^{H}<\ldots<Q_{n}^{H}<Q_{n+1}^{H}=Q^{L}$ is a sufficient condition for optimal solution, i.e., the manufacturer would ask all the suppliers to produce different amount of components when their cost type is high, which are strictly lower than their low costs quantity. When sufficient condition is violated, some neighboring suppliers would produce same amount of product. Thus, the optimal solution to problem (7) can be generally caracterized as a $m$-group solution, $m \leq n$.

For each solution group, we denote the head pointer and tail pointer for each group as $l_{i}, u_{i}, \forall i=1, \ldots, m$. Therefore, $l_{1}=1, l_{i} \leq u_{i}, l_{i}=u_{i}-1+1, u_{m}=n+1$. Suppliers in the same group has identical high cost procurement quantity, that is

$$
Q_{l_{i}, u_{i}}^{*}=Q_{k}^{H}, \forall l_{i} \leq k \leq u_{i}
$$

We initially put each $Q_{i}^{H}$ into a separate group, so we have $m=n+1$ groups. If $Q_{l_{i}, u_{i}}^{*}<Q_{l_{i+1}, u_{i+1}}^{*}$ is satisfied for all $i=1, \ldots, m-1$, then we reach a unique solution. The algorithm is formalized below:

Algorithm 1 Finding the optimal solution to problem (7)

STEP 1: Initialization. Denote $m=n+1, l_{i}=i, u_{i}=i$.

STEP 2: Compute optimal procurement quantity $Q_{l_{i+1}, u_{i+1}}^{*}$ for each group

STEP 3: Traversal and Numerical correction. IF $Q_{l_{i}, u_{i}}^{*}<Q_{l i+1, u_{i+1}}^{*}, \forall i=1, \ldots, m-1$, THEN $Q_{k}^{H}=Q_{l_{i}, u_{i}}^{*}$. OTHERWISE:

- let $k$ be the smallest index $i$ which satisties $Q_{l_{i}, u_{i}}^{*} \geq Q_{l_{i+1}, u_{i+1}}^{*}$. We merge group $k$ and group $k+1$.

- $u_{k} \leftarrow u_{k+1}$

- $l_{j} \leftarrow l_{j+1}, u_{j} \leftarrow u_{j+1}, j=k+1, \ldots, m-1$

- $m \leftarrow m-1$

This algorithm significantly reduces the complexity to $o(n)$ from which the original problem is $o\left(2^{n}\right)$. That means the manufacturer's problem can be solved under linear time complexity.

The main reason for the reduction of problem complexity is that theorem 1 guarantees suppliers is well arranged based on their optimal 1-supplier procurement quantity. We combine algorithm above with supplier's profit function, and the simplified function can be written as follows:

$$
\Pi_{i}^{H}=0, \forall i=1, \ldots, n \quad \Pi_{i}^{L}=Q_{i}^{H}\left(c_{i}^{H}-c_{i}^{L}\right), \forall i=1, \ldots, n
$$

Under cost information asymmetry, high cost suppliers $i(i=1, \ldots, n)\left(c_{i}=c_{i}^{H}\right)$ always generate zero profit, whereas low cost suppliers could have positive gains. Meanwhile, low cost suppliers' final profits depend on their high cost order quantity and difference between two cost types.

\section{Parameter Analysis}

In this section, we study the impact of market demand and final product price on manufacturer's profit function, which leads to the value of cost information assessment. We define manufacturer's information value as $V=\tilde{\Pi}-\Pi$ . Because manufacturer acquires full profit under symmetric information, $\Pi<\tilde{\Pi}$. Profit function for each supplier $i$ is $V_{i}=-\Pi_{i}=-p_{i}^{L} \Pi_{i}^{L}, \Pi_{i}$ is supplier's ex ante profit. Supply chain's information value is denoted as $V_{T}=V+\sum_{i=1}^{n} V_{i}$.

\subsection{Market Demand}

We first analyze how market demand distribution affect 
the optimal contract. We assume the demand follows a normal distribution, i.e., $D \sim N(\mu, \sigma)$. The probability of demand being negative is negligible, thus $\mu>>\sigma>0$. We derive the following results regarding the change of average demand, .

Proposition 1 If $D \sim(\mu, \sigma)$, then

- $\boldsymbol{\Pi}$ and $\tilde{\boldsymbol{\Pi}}$ are increasing in $\mu$.

- $V, V_{T}$ and $\boldsymbol{\Pi}_{i}, \forall i=1, \ldots, n$ are increasing in $\mu$.

It can be interpreted as follows. First, demand expansion would increase supplier's order quantity. $\forall i=1, \ldots, n$, $Q_{i}^{H}$ and $Q_{i}^{L}=Q^{L}$ constantly increase when $\mu$ increases. Under asymmetric information, if average market demand increases, manufacturer would definitely urge suppliers to produce more components. Suppliers' profit would also increase because $\Pi_{i}=p_{i}^{L} \Pi_{i}^{L}=p_{i}^{L} Q_{i}^{H}\left(c_{i}^{H}-c_{i}^{L}\right), \forall i=1, \ldots, n$. Higher average demand generates more information rent for suppliers.

Second, manufacturer's profit would also increase. Manufacturers can earn greater profits in the face of higher market demand in both the symmetric and asymmetric information scenarios. With symmetric information, the manufacturer captures all benefits in the supply chain, so its profits must increase as the market demand increases. Under asymmetric information, more sales volume also leads to higher returns. Still, manufacturers need to pay more information rent for suppliers, so the yields to manufacturers under asymmetric information rise less with average demand than under symmetric information.

Third, information value for whole supply chain would increase. Suppliers acquire higher information rent while information value increases even faster for manufacturer. Thus, the total information value increases.

\subsection{Final Product Price}

Assume manufacturer sales the product at price $\hat{r}$, which is increased from $r$, when other factors remain the same. According to high cost order quantity $q_{i}^{0}=\bar{F}^{-1}\left(\frac{c_{i}^{H}-p_{i}^{L} c_{i}^{L}}{p_{i}^{H} r}\right)$ each supplier increases their order quantity when final price increases, $q_{k}^{0} \leq \hat{q}_{k}^{0}, k=1, \ldots, n$. Therefore, we have $\hat{Q}_{n+1}^{H}=\hat{Q}^{L} \geq Q^{L}=\left\{Q_{n+1}^{H}\right.$. We can have the following result:

$$
\Pi\left(\mathrm{Q}_{1}^{H}, \ldots, \mathrm{Q}_{n+1}^{H} \mid \hat{r}\right)^{\hat{r}>r} \geq \Pi\left(\mathrm{Q}_{1}^{H}, \ldots, \mathrm{Q}_{n+1}^{H} \mid r\right)=\Pi
$$

We can prove that $\tilde{\boldsymbol{\Pi}} \geq \boldsymbol{\Pi}$.Similarly, $\widehat{\tilde{\boldsymbol{\Pi}}} \geq \tilde{\boldsymbol{\Pi}}$. It can be interpreted as follows: First, When the selling price of the final product increases, manufacturers will expect suppliers to make more parts because at that point the expected loss from out-of-stocks increases, and the unit storage costs from inventory backlogs remain unchanged.
At higher unit product prices, both in the case of information symmetry and in the case of information asymmetry, the manufacturer's marginal returns increase, and thus the returns under the manufacturer's optimal contract.

At the same time, when the selling price of a product raises, the manufacturer's earnings are raised more under symmetric information than under asymmetric information, because the manufacturer always gets the full benefit of the supply chain.

\section{Conclusion}

In order to reduce costs and increase revenues, many companies in the industrial sector will try to transform themselves into manufacturers who are only responsible for final assembly and outsource the production of components to upstream suppliers. The transformation of such business functions has gradually become a trend in the transformation of MNCs. However, upstream suppliers are often reluctant to work very deeply with suppliers due to their financial and target independence from the manufacturer, so manufacturers often incur additional costs in contracting to ensure that contracts run smoothly. This additional expenditure is the main objective of the study in this paper. In this paper's hypothesis, suppliers have full knowledge of their cost structure as private information, while downstream manufacturers only have subjective judgments about their cost structure and do not know accurate information.

The idea behind this paper's solution to information asymmetry is to design a contracting approach for manufacturers, in which manufacturers gain maximum benefit by giving suppliers a menu of contracts to choose from to understand the types of costs to suppliers. The contract menu presented in this article contains two parameters, the quantity purchased and the price paid by designing efficient algorithms to solve the contract menu for each vendor in an ideal state. By applying the contract menu design approach in this article, companies can effectively reduce unnecessary costs in supply chain collaboration and increase their revenue.

By analyzing the model parameters, this paper has further explored the specific impact that contract design solutions can have on the overall effectiveness of the supply chain under cost information asymmetry. For example, when market demand increases or when the selling price of a final product increases, information about a supplier's cost structure becomes more valuable to the manufacturer, and therefore the manufacturer is more motivated to obtain specific information about the supplier's cost structure.

This paper examines the deficiencies in contract de- 
sign mechanisms for manufacturers facing upstream information asymmetry. Information types will be further expanded in future studies. First, assumptions made in this paper about the types of supplier costs are based on discrete distributions, and future research can further make assumptions about different distributions to match the realities of the firm more closely. Second, this paper assumes that suppliers can fully meet manufacturers' ordering needs without considering the potential for supply disruptions in the event of unknown supply chain risks. The next study will further introduce supply disruption risk as a consideration in order to investigate the supply chain robustness approach. Finally, this paper focuses on the cost structure of production as a manufacturer contract design strategy under private information, and the mechanisms by which other different types of information influence manufacturer contract design can be further studied in the future.

\section{References}

[1] Iyer, V. A., B. L. Schwarz, A. S. Zenios. A principal-agent model for product specification and production[J]. Manage Sci,2005(01): 106-119.

[2] Zhuoxin Li, Stephen M. Gilbert, Guoming Lai. Supplier Encroachment Under Asymmetric Informa-
tion[J]. Management Science,2014(02):449-462.

[3] Song Huang, Xu Guan, Ying-Ju Chen Retailer Information Sharing with Supplier Encroachment[J]. Production and Operations Management 2018(06):11331147.

[4] Zhuoxin Li, Stephen M. Gilbert, Guoming Lai. Supplier Encroachment as an Enhancement of a Hindrance to Nonlinear Pricing[J]. Production and Operations Management,2014(01):89-109.

[5] Anil Arya, Brian Mittendorf, David E. M. Sappington. The Bright Side of Supplier Encroachment[J]. Marketing Science, 2007(05):651-659.

[6] Wang, Y. Joint pricing-production decisions in supply chains of complementary products with uncertain demand[J]. Oper. Res. 2006: 1110-1127.

[7] Fang, X., Y. Wang. A model for partial product complementarity and strategic production decisions under demand uncertainty[J]. Prod. Oper. Manag. 2010(03): 322-342.

[8] Babich, V., C. S. Tang. Managing opportunistic supplier product adulteration: Deferred payments, inspection. And combined mechanisms[J]. Manuf. Serv. Oper. Manag. 2010(02): 301-314.

[9] SH Xu, T Yao. Strategic Outsourcing Contract Participation and Selection under Cost Uncertainty $[\mathrm{J}]$. 2013. 